\title{
Seismology of kink oscillations in coronal loops: Two decades of resonant damping
}

\author{
Marcel Goossens \\ Centre Plasma Astrophysics, Katholieke Universiteit Leuven, Leuven, B-3001, Belgium \\ email: Marcel.Goossens@wis.kuleuven.be
}

\begin{abstract}
.
The detection of rapidly damped transverse oscillations in coronal loops by Aschwanden et al. (1999) and Nakariakov et al. (1999) gave a strong impetus to the study of MHD waves and their damping. The common interpretation of the observations of these oscillations is based on kink modes. This paper reviews how the observed period and damping time can be reproduced by MHD wave theory when non-uniform equilibrium models are considered that have a transversal variation of the local Alfven velocity. The key point here is that resonant absorption cannot be avoided and occurs as natural damping mechanism for kink waves in non-uniform equilibrium models. The present paper starts with work by Hollweg \& Yang (1988) and discusses subsequent developments in theory and their applications to seismology of coronal loops. It addresses the consistent use of observations of periods and damping times as seismological tools within the framework of resonant absorption. It shows that within the framework of resonant absorption infinitely many equilibrium models can reproduce the observed values of periods and damping times.
\end{abstract}

Keywords. MHD waves, coronal loops, resonant damping.

\section{Introduction}

In my view the detection of heavily damped transverse oscillations in coronal loops by Aschwanden et al. (1999) and Nakariakov et al. (1999) was the real start of coronal seismology. These oscillations have periods of the order of $\simeq 2-10$ minutes and comparatively short damping times of the order of $\tau_{d} \simeq 3-20$ minutes. The common interpretation of these oscillations is that they are fast standing kink mode oscillations. Kink oscillations are singled out because they have azimuthal wave $m=1$ so that the axis of the loop is displaced. The big puzzle was the cause of their rapid damping

A possible mechanism for explaining the observed rapid damping might already have been known in the 1980s. In 1988 Hollweg \& Yang (1988) published a paper entitled Resonance Absorption of Compressible Magnetohydrodynamic Waves at Thin "Surfaces". In that paper Hollweg \& Yang (1988) studied MHD surface waves in a planar geometry with a thin transitional layer of thickness $l$ as a possible means for heating the solar corona by MHD waves. A plasma system with two infinite uniform plasmas separated by a true discontinuity where the equilibrium plasma quantities $f$ such as e.g. density $\rho$ change discontinuously supports the classic surface wave with its square of frequency $\omega$ equal to the weighted mean of the squares of the local Alfvén frequencies

$$
\omega^{2}=\omega_{k}^{2}=\frac{\rho_{i} \omega_{A, i}^{2}+\rho_{e} \omega_{A, e}^{2}}{\rho_{i}+\rho_{e}}
$$

$\omega_{A}$ is the local Alfvén frequency defined as

$$
\omega_{A}=k_{\|} v_{A}, \quad v_{A}=B / \sqrt{\mu \rho}
$$


$k_{\|}$is the wave number along the equilibrium magnetic field and $v_{A}$ is the local Alfvén velocity. When the true discontinuity is replaced by a continuous variation of the local Alfvén frequency the ideal MHD equations are characterized by a singularity at position where $\omega=\omega_{A}$. A possible way to avoid this mathematical singularity is to include dissipation in the MHD equations. However, this comes with a price, the dissipative MHD equations are definitely more complicated to handle than their ideal counterparts. Hollweg \& Yang (1988) adopted a simple scheme that enabled them to include dissipation in their analysis without having to solve the dissipative MHD wave equations. Their trick was to assume that the Eulerian perturbation of total pressure, $P^{\prime}$ is constant across the dissipative layer

$$
\left[P^{\prime}\right]=0
$$

for resonant Alfvén waves. Here $[f]$ denotes the jump the quantity $f$ undergoes when crossing the dissipative layer. In their analysis the non-uniform transition layer, cfr. thin surface, coincides with the dissipative layer. In doing so Hollweg \& Yang (1988) had to solve the ideal MHD wave equations in two uniform plasmas and connect the solutions over the dissipative layer and also the thin non-uniform transitional layer by the use of (1.3). Hollweg \& Yang (1988) concentrated on propagation nearly perpendicular to the equilibrium magnetic field. Damping of the surface wave is seen as energy being transferred from the MHD wave to heating of the plasma.

For a linear variation of the equilibrium density we can write Eq. 69 of Hollweg \& Yang (1988) as

$$
\omega \tau_{d}=\frac{8\left(\rho_{i}+\rho_{e}\right)}{\pi(k l)\left|\rho_{i}-\rho_{e}\right|}
$$

In this equation $\omega$ is the frequency, $\tau_{d}$ is the decay time, $k \approx k_{\perp}$ is the wave number and $l$ is the width of thin non-uniform layer. Note that (1.4) is not given in the paper by Hollweg \& Yang (1988). In their discussion Hollweg \& Yang (1988) say that "Even though the above analysis assumes slab geometry, we shall here illustrate the foregoing ideas using numbers appropriate to solar coronal active regions loops, for which toroidal or cylindrical geometry might be more appropriate." Let us use the following prescriptions to transform (1.4) obtained for planar geometry into a corresponding expression for cylindrical geometry $\left(k_{\perp}\right.$ is the wave number in the direction in the magnetic surfaces perpendicular to the magnetic field lines) :

$$
k_{\perp}=\frac{m(=1)}{R}, \quad k_{\|}=\frac{\pi}{L}, \quad k_{\|} \ll k_{\perp} \approx k, R=\text { radius, } L=\text { loop length }
$$

This enables us to rewrite Eq. 69 of Hollweg \& Yang (1988) as

$$
\frac{\tau_{d}}{\text { Period }}=\frac{4}{\pi^{2}} \frac{R}{l} \frac{\rho_{i}+\rho_{e}}{\rho_{i}-\rho_{e}}
$$

Again note that (1.5) is not given in the paper by Hollweg \& Yang (1988). Also, note that (1.5) is Eq. 79b of Goossens et al. (1992), but we shall come back to that in subsection 4.4. Hollweg \& Yang (1988) take the numerical example

$$
l / R=2 / 5, \quad \rho_{i} / \rho_{e}=3
$$

and find that

$$
\frac{\tau_{d}}{\text { Period }}=\frac{20}{\pi^{2}} \approx 2
$$

This result led Hollweg \& Yang (1988) to conclude that " the waves are very effectively 
damped with an e-folding time of only two wave periods. This rapid damping of the surface wave indicates that resonance absorption is a viable candidate for coronal heating."

In retrospect it is difficult to understand that in 1999, when the rapidly damped kink oscillations were detected in TRACE observations, nobody linked the fast damping found by Hollweg \& Yang (1988) in the context of coronal heating to the observed damping of the TRACE loop oscillations. When I tried to understand the Hollweg \& Yang (1988) paper in the summer of 1989 I was puzzled by following questions. How relevant are results obtained for planar geometry for cylindrical coronal loops? Is the ad hoc conservation law (1.3) correct and is it possible to put it on a solid mathematical basis? What about the thin transitional layer that coincides with the dissipative layer? In view of the large values of the magnetic Reynolds number in the solar corona, this transitional layer must be really very thin since $l / R \sim R_{m}^{-1 / 3} \approx 10^{-4}$ while the numerical example of Hollweg \& Yang (1988) adopts $l / R=2 / 5$. What about the Taylor expansion of dispersion relation which is strictly speaking only valid if $\left|\omega_{i}\right| /\left|\omega_{R}\right| \ll 1$ while Hollweg \& Yang (1988) find that $\tau_{d}$ /Period $\approx 2$ clearly violating that assumption.

\section{MHD waves in 1-D cylindrical equilibrium models}

\subsection{Equilibrium model}

The equilibrium model is a flux tube in static equilibrium. We use cylindrical coordinates $r, \varphi, z$. The equilibrium quantities $\vec{B}=\left(0, B_{\varphi}(r), B_{z}(r)\right), p(r)$ and $\rho(r)$ depend on the radial distance to the axis of the cylinder. The equilibrium quantities satisfy the wellknown radial force balance equation $\frac{d}{d r}\left(p+\frac{B^{2}}{2 \mu}\right)=-\frac{B_{\varphi}^{2}}{\mu r}, \quad B=\left(B_{\varphi}^{2}+B_{z}^{2}\right)^{1 / 2}$.

\subsection{MHD waves equations and coupling between MHD waves}

On the equilibrium state we superimpose linear motions. The time dependence of these linear motions is prescribed as $\exp (-i \omega t)$. As said before, $\omega$ is the frequency. In the driven problem it is prescribed, in the eigenvalue problem it is a quantity to be determined. Since the background is independent of $(\varphi, z)$ we can Fourier-analyze the perturbed quantities with respect to these two ignorable variables and put them proportional to $\exp \left(i\left(m \varphi+k_{z} z\right)\right) \cdot m, k_{z}=$ are the azimuthal and axial wave numbers and the wave vector in the magnetic surfaces is $\vec{k}=\left(0, m / r, k_{z}\right)$. When MHD waves are studied in 2-dimensional equilibrium models with longitudinal stratification of density, density is also a function of $z, \rho(r, z)$. In that case it is no longer possible to describe the MHD waves with one value of $k_{z}$ (see e.g. Andries et al. (2005a)).

In what follows $\vec{\xi}$ is the Lagrangian displacement, $P^{\prime}$ is the Eulerian perturbation of total pressure, $\xi_{\perp}, \xi_{\|}$are the components in magnetic surfaces perpendicular/parallel to the magnetic field lines. To understand the physics of the waves it is important to note that $\xi_{\perp}$ characterizes the Alfvén waves, $\xi_{r}$ characterizes the fast magneto-sonic waves and $\xi_{\|}$characterizes the slow magneto-sonic waves. The equations for the linear MHD waves on a 1-dimensional cylinder are (see e.g. Appert et al. (1974); Sakurai et al. (1991a); Goossens et al. (1992), Goossens et al. (1995))

$$
\begin{aligned}
D \frac{d\left(r \xi_{r}\right)}{d r} & =C_{1} r \xi_{r}-C_{2} r P^{\prime}, \\
D \frac{d P^{\prime}}{d r} & =C_{3} \xi_{r}-C_{1} P^{\prime}, \\
\rho\left(\omega^{2}-\omega_{A}^{2}\right) \xi_{\perp} & =\frac{i}{B} C_{A},
\end{aligned}
$$




$$
\rho\left(\omega^{2}-\omega_{C}^{2}\right) \xi_{\|}=\frac{i f_{B}}{B} \frac{v_{S}^{2}}{v_{S}^{2}+v_{A}^{2}} C_{S},
$$

The quantities $D, C_{1}, C_{2}, C_{3}, C_{A}$ and $C_{S}$ that appear in equations (2.1) can be found in e.g. Sakurai et al. (1991a); Goossens et al. (1992), Goossens et al. (1995), and by Tirry \& Goossens (1996). The equations for MHD waves in a cylindrical plasma with an equilibrium flow can be found in e.g. Goossens et al. (1992), Erdélyi et al. (1995) and Erdélyi (1997). The Cartesian version (2.1) with a horizontal magnetic field and gravity in the vertical direction are given in e.g. Tirry et al. (1998b) and Pintér et al. (2007). Finally, the coefficients for a uniformly twisted magnetic flux tube are in Erdélyi \& Fedun (2006, 2007).

In view of what follows it is instructive to list the coefficient function $D$ and the coupling factor $C_{A}$ :

$$
D=\rho\left(v_{S}^{2}+v_{A}^{2}\right)\left(\omega^{2}-\omega_{A}^{2}\right)\left(\omega^{2}-\omega_{C}^{2}\right), \quad C_{A}=g_{B} P^{\prime}-\frac{2 f_{B} B_{\varphi} B_{z} \xi_{r}}{\mu r}
$$

with

$$
\begin{aligned}
& f_{B}=\vec{k} \cdot \vec{B}=k_{z} B_{z}+\frac{m B_{\varphi}}{r}, \quad g_{B}=\frac{m B_{z}}{r}-k_{z} B_{\varphi} \\
& \omega_{A}^{2}=\frac{(\vec{k} \cdot \vec{B})^{2}}{\mu \rho}, \quad \omega_{C}^{2}=\frac{v_{S}^{2}}{v_{S}^{2}+v_{A}^{2}} \omega_{A}^{2}, \quad v_{S}^{2}=\frac{\gamma p}{\rho}, \quad v_{A}^{2}=\frac{B^{2}}{\mu \rho}
\end{aligned}
$$

$\omega_{A}$ and $\omega_{C}$ are the local Alfvén and the local cusp frequency; $v_{S}$ and $v_{A}$ are the local speed of sound and the local Alfvén velocity. In the approximation of a pressureless plasma $v_{S}=0$ and $\omega_{C}=0$. Hence, the slow magneto-sonic waves are removed from the analysis and $\xi_{\|}=0$. For the present discussion there are two points to be made. The first point has to do with the function $C_{A}$. It couples the equations for the magneto-sonic waves, with that for the Alfvén waves. In general $C_{A} \neq 0$ and the fast magneto-sonic waves and the Alfvén waves are coupled and do interact. There is one exception. In case of an equilibrium with a straight field $\left(B_{\varphi}=0\right)$ the coupling factor $C_{A}=0$ for $m=0$ so that there is no interaction between the fast sausage waves and the torsional Alfvén waves since they have $m=0$. In short: there is always interaction between fast magneto-sonic waves and Alfvén waves except for a straight field and $m=0$.

For a straight field $\vec{B}=B(r) \overrightarrow{1}_{z}$ the magnetic surfaces are cylinders: $r=$ constant and the $\varphi$ - direction and $z$-direction are the directions in the magnetic surfaces respectively perpendicular and parallel to the equilibrium magnetic field. The $r$-direction is normal to the magnetic surfaces. Consequently $\xi_{\varphi}$ characterizes the Alfvén waves, $\xi_{r}$ characterizes the fast waves and $\xi_{z}$ characterizes the slow waves. The equations for the linear MHD waves on a 1-dimensional cylinder with a straight field are

$$
\begin{aligned}
D \frac{d\left(r \xi_{r}\right)}{d r} & =-C_{2} r P^{\prime}, \\
\frac{d P^{\prime}}{d r} & =\rho\left(\omega^{2}-\omega_{A}^{2}\right) \xi_{r} \\
\rho\left(\omega^{2}-\omega_{A}^{2}\right) \xi_{\varphi} & =\frac{i m}{r} P^{\prime} \\
\rho\left(\omega^{2}-\omega_{C}^{2}\right) \xi_{z} & =i k_{z} \frac{v_{S}^{2}}{v_{S}^{2}+v_{A}^{2}} P^{\prime} .
\end{aligned}
$$

These equations clearly show that for $m=0$ the Alfvén waves and the magneto-sonic 
waves do not interact, while for $m \neq 0$ they do interact. For more details in a twisted tube see Erdélyi \& Fedun (2006, 2007).

\section{Resonant Alfvén waves in 1-D cylindrical equilibrium models}

The second observation to be made has to do with the coefficient function $D$. In a non-uniform plasma $\omega_{A}(r)$ varies with position $r$ and defines a continuous range

$$
\left[\min \omega_{A}(r), \max \omega_{A}(r)\right]
$$

known as the Alfvén continuum (we forget about the slow continuum as it does not exist when we adopt the approximation of a pressureless plasma as we do in the present analysis). $D=0$ at the position $r_{A}$ where $\omega=\omega_{A}\left(r_{A}\right)$. Hence $r_{A}$ is a mobile regular point of (2.1) and (2.4). The solutions that correspond to a frequency in the Alfvén continuum have singular spatial functions in ideal MHD and are known as resonant Alfvén continuum waves (see e.g Appert et al. (1974), Chen \& Hasegawa (1974), Goedbloed (1983)). The dispersion relation for Alfvén waves is locally satisfied on each magnetic surface and each magnetic surface oscillates at its own Alfvén continuum frequency. The singular (non-square integrable) spatial solutions in ideal MHD $\left(s=r-r_{A}\right)$ are a logarithmic singularity and a jump for $P^{\prime}, \xi_{\|}, \xi_{r}$ and a $1 / s$-singularity and a $\delta(s)$ - contribution for $\xi_{\perp}$. Hence the dominant dynamics is in the component in the magnetic surfaces perpendicular to the magnetic field lines: $\xi_{\perp}$.

Now recall that there is always interaction between fast magneto-sonic waves and Alfvén waves except for a straight field and $m=0$. For $m=0$ and $\vec{B}=B \overrightarrow{1}_{z}$ the (real) eigenvalues of discrete (fast) eigenmodes can lie in the Alfvén continuum, but there is no coupling. For $m \neq 0$ the discrete (fast) eigenmodes with an eigenfrequency in the Alfvén continuum couple to a local Alfvén continuum eigenmode and produce quasi-modes. The energy of the global eigenmode is transferred to local continuum waves. Large (infinite) gradients are built up near the resonances so that dissipative effects become important and have to be taken into account.

The driven problem for resonant Alfvén waves was studied by Sakurai et al. (1991a), Goossens et al. (1992), Goossens et al. (1995), and Erdélyi \& Goossens (1996) for 1dimensional cylindrical equilibrium models and by Tirry \& Goossens (1995) for 2-dimensional equilibrium models. The eigenvalue problem was investigated by Tirry \& Goossens (1996), Tirry et al. (1998a) and Tirry et al. (1998b) for 1-dimensional cylindrical and Cartesian models respectively. Here we recall the essential steps in Goossens et al. (1995). The analysis is restricted to linear motions that correspond to resonant Alfvén waves in ideal MHD. This allows for a first simplification of the original linear dissipative MHD equations. Subsequently the coefficient functions are replaced by their linear Taylor series expansions around the ideal Alfvén resonance $\left(r=r_{A}, s=0\right)$. This allows for obtaining a simplified set of dissipative MHD equations valid in the interval $\left[-s_{A}, s_{A}\right]$ around the point of resonance. These simplified dissipative MHD equations are free from singularities. The solutions to these equations are finite everywhere but have steep gradients in the vicinity of the ideal resonant point. The dissipative layer where dissipation is important has a width that is measured by the quantity $\delta_{A}$ given by (see e.g. Kappraff \& Tataronis (1977); and Hollweg \& Yang (1988))

$$
\delta_{A}=\left(\frac{\omega \eta}{|\Delta|}\right)^{1 / 3}, \Delta=\frac{d}{d r}\left(\omega^{2}-\omega_{A}^{2}\right)
$$

In view of the very large values of the magnetic Reynolds number $R_{m}$ in the solar 
corona it follows that $s_{A} / \delta_{A} \gg 1$. This inequality is important because it guarantees that the simplified dissipative MHD equations are valid in the dissipative layer and in two overlap regions to the left and right of the dissipative layer where ideal MHD is valid. It is convenient to introduce the scaled variable $\tau=s / \delta_{A}$ which is of order 1 in the dissipative layer, but $s \rightarrow \pm s_{A}$ corresponds to $\tau \rightarrow \pm \infty$. The simplified dissipative linear MHD equations are a set of two ordinary differential equations of third order for the variables $\xi_{r}$ and $P$ and an ordinary differential equation of second order for the variable $\xi_{\perp}$. The first step for solving this set of ordinary differential equations, is to obtain the differential equation that determines the coupling function $C_{A}$. The bounded solution for $C_{A}(\tau)$ is amazingly simple

$$
C_{A}(\tau)=\text { constant }
$$

This is the fundamental conservation law for resonant Alfvén waves in dissipative MHD. A similar conserved quantity was found by Erdélyi (1997) for resonant slow waves. Once this conservation law is established the solutions for $\xi_{r}, P^{\prime}$, and $\xi_{\perp}$ that remain finite for $|\tau| \rightarrow \infty$ can be determined. Sakurai et al. (1991a) obtained solutions in terms of double integrals of Hankel functions of complex argument of order 1/3. A set of more compact solutions can be obtained by the aid of the $F$ and $G$ functions (see e.g. Goossens et al. (1995), Goossens \& Ruderman (1995), Tirry \& Goossens (1996), Tirry et al. (1998b) and Pintér et al. (2007)):

$$
\begin{aligned}
\xi_{r} & =-\frac{g_{B} C_{A}}{\rho B^{2} \Delta} G(\tau)+C_{\xi}, \quad P^{\prime}=-\frac{2 f_{B} B_{\varphi} B_{z} C_{A}}{\rho B^{2} \mu r \Delta} G(\tau)+C_{P}, \\
\xi_{\perp} & =\frac{C_{A}}{\delta_{A}|\Delta| \rho B} F(\tau)
\end{aligned}
$$

$C_{\xi}$ and $C_{P}$ are constants of integration. The functions $F$ and $G$ are

$$
\begin{aligned}
& G(\tau)=\int_{0}^{\infty}\left(e^{i u \operatorname{sign}(\Delta) \tau-\Lambda u}-1\right) \frac{e^{-\frac{u^{3}}{3}}}{u} d u, \\
& F(\tau)=\int_{0}^{\infty} e^{i u \operatorname{sign}(\Delta) \tau-\Lambda u} e^{-\frac{u^{3}}{3}} d u
\end{aligned}
$$

The quantity $\Lambda$ is zero in case of the driven problem with a prescribed real valued frequency $\omega$; in case of the eigenvalue problem it is related to the imaginary part of the frequency as $\Lambda=-\frac{2 \omega_{R} \omega_{I}}{\delta_{A}|\Delta|} \operatorname{In}$ (3.4) the $G$ and $F$ functions recover the ideal singular behaviour but only sufficiently far away from the ideal resonant position. (3.4) combined with(3.5) give the solutions in the dissipative layer and in two overlap regions to the left and the right of the dissipative layer. In the overlap regions the motions are accurately described by the ideal MHD equations also. Hence, when we are interested in the details of the behaviour of the waves in the dissipative layer then we have to use (3.4) combined with(3.5). However, if we are primarily concerned with the global solutions then we can suffice with jump relations that tell us how to connect the solution to the left of the dissipative layer to that of the right to the dissipative layer. From (3.3) it follows that

$$
\left[C_{A}\right]=0
$$

From Appendix B of Goossens et al. (1995) we learn that $[G]=i \pi$ Hence the jumps in 
$\xi_{r}$ and $P^{\prime}$ are

$$
\left[\xi_{r}\right]=-i \pi \frac{g_{B} C_{A}}{\rho B^{2}|\Delta|}, \quad\left[P^{\prime}\right]=-i \pi \frac{2 f_{B} B_{\varphi} B_{z} C_{A}}{\rho B^{2} \mu r_{A}|\Delta|}
$$

The jumps and conservation law were first derived by Sakurai et al. (1991a) for the driven problem and by Tirry \& Goossens (1996) for the eigenvalue problem. The jumps are independent of dissipation and hence the damping is independent of dissipation. The jumps were in first instance used by Sakurai et al. (1991b) to compute the absorption of acoustic oscillations in sunspots. They were used by Goossens \& Hollweg (1993) to determine the optimal conditions for absorption of driven resonant Alfvén waves and implemented in a simple numerical scheme for the computation of driven resonant Alfvén waves by Stenuit et al. (1995). The jumps and conservation laws in 1-d stationary equilibrium models were obtained in ideal MHD by Goossens et al. (1992) and in dissipative MHD by Erdélyi et al. (1995), Erdélyi (1997).

For a straight field $B_{\varphi}=0$ the jump relations (3.6) and (3.7) take a simpler form

$$
\left[P^{\prime}\right]=0, \quad\left[\xi_{r}\right]=-i \pi \operatorname{sign}(\omega) \frac{m^{2} / r^{2}}{\rho|\Delta|} P^{\prime}, \quad[\text { Flux } E]=-\frac{\pi|\omega| m^{2}}{2 \rho|\Delta|}\left|P^{\prime}\right|^{2} .
$$

[FluxE] is the jump of energy when crossing the dissipative layer. In a static equilibrium this jump is always negative, meaning that the dissipative layer is a sink for the energy of the wave so that the wave gets damped. In equilibrium models with flow the quasimodes can become overstable as shown by e.g. Andries et al. (2000), Andries \& Goossens (2001a), Andries \& Goossens (2001b) in solar wind, and Taroyan \& Erdélyi (2002), Erdélyi \& Taroyan (2003) and Taroyan \& Erdélyi (2003) in magnetospheric applications. Note that in an equilibrium with a straight magnetic field the Eulerian perturbation of total pressure does not undergo a jump when crossing the dissipative layer. Note that [FluxE] is proportional to $\left|P^{\prime}\right|^{2}$ so that the amount of energy absorbed is strongly dependent on the absolute value of the pressure perturbation.

In addition, for an equilibrium with a straight magnetic field, $\xi_{r}$ and FluxE do not jump, $\left[\xi_{r}\right]=0$ and $[$ FluxE] $=0$ for waves with $m=0$. Hence waves with $m=0$ are not resonantly absorbed in an equilibrium with a straight field. This comes as no surprise since torsional Alfvén waves and sausage fast waves are not coupled in an equilibrium with a straight field. Here is a good point to go back to the 1980s. The result (3.8), i.e. $\left[P^{\prime}\right]=0$ means that the assumption used by Hollweg \& Yang (1988) is correct. In addition, there is no need for the non-uniform transitional layer to coincide with the dissipative layer. The assumption that the non-uniform transitional layer coincides with the dissipative layer is used to connect analytical solutions for a uniform plasma to the left of the transitional layer to those to the right of the dissipative layer. In what follows we refer to this approximation as the thin boundary (TB) approximation.

\section{Where is the damped eigenmode?}

\subsection{Quasi-modes in non-uniform plasma}

The linear spectrum of MHD waves of a non-uniform static plasma equilibrium consists of discrete (fast and slow magneto-sonic and Alfvén ) eigenmodes and continuum Alfvén eigenmodes. Remember that there is always interaction between fast magneto-sonic waves and Alfvén waves except for a straight field and $m=0$. This means that for an equilibrium with a straight magnetic field the real eigenvalues of discrete fast sausage eigenmodes can lie in the continuum of the torsional Alfvén continuum eigenmodes, but there is no coupling and hence no damping. On the other hand for $m \neq 0$ the discrete fast 
eigenmodes with an eigenfrequency in the Alfvén continuum couple to a local Alfvén continuum eigenmode to form a damped quasi-mode. Energy of the global eigenmode is transferred to local continuum waves. For a static equilibrium there is always extraction of wave energy at the resonances and the quasi-modes are damped as can seen from the expression (3.8) for [FluxE]. Tirry \& Goossens (1996) gave a nice illustration of this transformation of undamped sausage fast magneto-sonic eigenmodes with frequencies in the Alfvén continuum into kink fast magneto-sonic eigenmodes damped by resonant absorption. These authors considered a pressureless cylindrical plasma equilibrium with a straight magnetic field and a density profile that varies with distance $r$ to the axis of the cylinder as $\rho(r)=\rho(0)\left[1+0.9 \exp \left(-(r / R)^{4}\right)\right]$. Tirry \& Goossens (1996) computed the first three sausage $(m=0)$ fast magneto-sonic eigenmodes of this equilibrium model for 31 equidistant values of $k_{z} R$ from 0 to 3 . For each $k_{z}$ the corresponding Alfvén continuum was computed. For $k_{z} R \geqslant 1$ the fundamental fast sausage mode has its frequency in the Alfvén continuum. This happens for the first overtone for $k_{z} R \geqslant 2$ and for the second overtone for $k_{z} R \geqslant 3$. Hence there are many sausage modes with frequencies in the Alfvén continuum. In order to see what happens with these modes when going from a sausage mode $m=0$ to a kink mode $m=1$ Tirry \& Goossens (1996) carried out a numerical experiment in which they varied the value of $m$ in a continuous manner from 0 to 1 . The outcome of this mathematical exercise is that the frequency becomes complex with a nonzero negative imaginary part and a real part that undergoes a shift. The excursion of the frequency in the complex plane has been computed for different values of the magnetic Reynolds number $R_{m}=10^{7}, 10^{8}, 10^{9}, 10^{10}$. The result is a numerical confirmation of the damping being independent of dissipation for sufficiently small dissipation as could have been predicted on the basis of the expression (3.8) for [FluxE]. The same conclusion was reached by Poedts \& Kerner (1991) in a study of the kink mode in a fusion related setup. Quasi-modes are the natural oscillation modes of a system. They combine properties of a localized resonant Alfvén wave and of a global fast eigenmode. They are damped due to resonant coupling and the damping is independent of dissipation for small dissipation. The role of the quasi-modes for coronal heating was studied by e.g. Poedts et al. (1989) and Poedts et al. (1990). The excitation of the quasi-modes by given initial perturbation was studied by e.g. Terradas et al. (2006) and Terradas et al. (2007).

\subsection{Non-leaky eigenmodes of uniform plasma cylinders}

The non-leaky eigenmodes of a uniform plasma cylinder with a constant axial magnetic field can be described by a 2 nd order ordinary differential equation for $P^{\prime}$

$$
\begin{gathered}
\frac{d^{2} P^{\prime}}{d r^{2}}+\frac{1}{r} \frac{d P^{\prime}}{d r}+\left\{\frac{\left(\omega^{2}-k_{z}^{2} v_{S}^{2}\right)\left(\omega^{2}-\omega_{A}^{2}\right)}{\left(v_{S}^{2}+v_{A}^{2}\right)\left(\omega^{2}-\omega_{C}^{2}\right)}-\frac{m^{2}}{r^{2}}\right\} P^{\prime}=0 \\
k_{\perp}^{2}= \pm \frac{\left(\omega^{2}-k_{z}^{2} v_{S}^{2}\right)\left(\omega^{2}-\omega_{A}^{2}\right)}{\left(v_{S}^{2}+v_{A}^{2}\right)\left(\omega^{2}-\omega_{C}^{2}\right)}
\end{gathered}
$$

is the square of the local radial wave number. The solutions to equation (4.1) can be obtained in terms of Bessel and Hankel functions. The dispersion relation follows from the requirements that the normal displacement and total pressure are continuous at the boundary $r=R$

$$
D\left(\omega, k_{z}, m\right) \equiv \frac{\xi_{r, i}(R)}{P_{i}^{\prime}(R)}-\frac{\xi_{r, e}(R)}{P_{e}^{\prime}(R)}=0
$$

When $k_{z}$ and $m$ are chosen the dispersion relation (4.2) has to be solved for the eigenvalue $\omega$. The equilibrium model has constant values of the local Alfvén frequency inside and outside the loop with a discontinuous variation at the boundary and MHD radiation 
is not considered (see e.g. Spruit(1982), Cally (1986), Stenuit et al. (1998) and Stenuit et al. (1999)). Hence, there is no damping. Edwin \& Roberts (1983) solve the dispersion relation (4.2) for photospheric flux tubes and for coronal loops. The present discussion focusses on the eigenmodes of coronal loops. For coronal loops $B_{i} \approx B_{e}, \quad \rho_{i}>\rho_{e}$ so that $v_{A, i}<v_{A, e}, \quad \omega_{A, i}^{2}<\omega_{A, e}^{2}$ On Figure 4 of Edwin \& Roberts (1983) it can be clearly seen that $v_{A, i}<\frac{\omega}{k_{z}}<v_{A, e}$ for kink waves. Hence

$$
\omega_{A, i}<\omega_{k i n k}<\omega_{A, e}
$$

In the thin tube (TT) approximation which assumes that the local radial wave length is much longer than the radius $R$ of the tube or equivalently that the radius of the loop is much shorter than the length $L$ of the loop $k_{\perp, i, e} R \ll 1, \quad R / L<<1$ we can use expansions of Bessel functions and find the following simple result

$$
\omega_{k i n k}^{2}=\frac{\rho_{i} \omega_{A, i}^{2}+\rho_{e} \omega_{A, e}^{2}}{\rho_{i}+\rho_{e}}
$$

Both (4.3) and (4.4) show that the frequency of the kink modes for a uniform coronal loop lies in between the internal and external value of the local Alfvén frequency. Hence when the true discontinuity is replaced with a continuous variation, the kink mode has its frequency in the Alfvén continuum and as a consequence will be damped by resonant absorption. We shall come back on this damping of kink modes by resonant absorption in a following subsection. First we shall look at a seismological application of (4.4).

\subsection{Seismology I}

Nakariakov (2000) and Nakariakov \& Ofman (2001) used TRACE observations of July 4, 1999 and the approximate expression for the frequency of the kink mode under the TT assumption to estimate the magnetic field strength. When we assume that $B_{i}=B_{e}=B_{0}$ (4.4) can be written as $\omega / k_{z} \approx\left(2 /\left(\mu\left(\rho_{e}+\rho_{i}\right)\right)\right)^{1 / 2} B_{0}$ which can be solved for $B_{0}$ giving

$$
B_{0} \approx \sqrt{2} \frac{L}{\text { Period }}\left(\mu\left(\rho_{i}+\rho_{e}\right)\right)^{1 / 2}
$$

If reliable estimates for density are available then (4.5) can be used to obtain an estimate for the magnetic field strength. The large uncertainties on density invariably lead to large error bars on $B$.

\subsection{Damped kink $(m \geqslant 1)$ quasi-mode (TTTB-approximation)}

Recall from (4.3) and (4.4) that the kink eigenmodes for a uniform coronal loop have their frequency in between the internal and external value of the local Alfvén frequency. Hence when the discontinuous transition $v_{A, i}$ to $v_{A, e}$ is replaced with a continuous variation, the kink mode has its frequency in the Alfvén continuum and as a consequence will be damped by resonant absorption. The classic kink mode is always a quasi-mode damped by resonant absorption. Its eigenvalues are complex, with their imaginary part independent of dissipation. Hence we write $\omega=\omega_{R}+i \gamma, \exp (-i \omega t)=\exp \left(-i \omega_{R} t\right) \exp (\gamma t)$. Goossens et al. (1992) confine the inhomogeneity to the interval

$$
b=R-l / 2 \leqslant r \leqslant a=R+l / 2
$$

and adopt the so-called thin boundary (TB) approximation: $l / R<<1$. The TB approximation of the dispersion relation is

$$
D\left(\omega, k_{z}, m\right) \equiv \frac{\xi_{r, i}(R)}{P_{i}^{\prime}(R)}-\frac{\xi_{r, e}(R)}{P_{e}^{\prime}(R)}+i \pi \operatorname{sign}(\omega) \frac{m^{2} / r_{A}^{2}}{\rho\left(r_{A}\right)|\Delta|}=0
$$


The third term in the left hand side of the dispersion relation (4.6) is due to the resonance. Goossens et al. (1992) solve the dispersion relation (4.6) in the TT approximation. We shall refer to the combination of these two approximations as TTTB. The result is

$$
\begin{aligned}
\omega_{R}^{2} & =\frac{\rho_{i} \omega_{A, i}^{2}+\rho_{e} \omega_{A, e}^{2}}{\rho_{i}+\rho_{e}}=\omega_{k}^{2} \\
\gamma & =-\frac{|m| \pi}{2 R} \frac{\rho_{1}^{2} \rho_{2}^{2}}{\rho\left(r_{A}\right)\left(\rho_{1}+\rho_{2}\right)^{3}} \frac{\left(\omega_{A, 2}^{2}-\omega_{A, 1}^{2}\right)^{2}}{\left|\omega_{k} \| \Delta\right|}
\end{aligned}
$$

This is equation 77 in Goossens et al. (1992). For equal and constant magnetic fields $B_{i}=B_{e}=B(4.7)$ reduces to

$$
\gamma=-\frac{\pi}{2 R} \frac{\rho_{A}\left|\omega_{k}\right|^{3}}{|\Delta|} \frac{\left(\rho_{2}-\rho_{1}\right)^{2}}{\left(\rho_{1}+\rho_{2}\right)^{3}}
$$

This is equation 56 of Ruderman \& Roberts (2002). Finally, for equal and constant magnetic fields $B_{i}=B_{e}=B$ and a linear variation of density, (4.7) takes the simple form

$$
\gamma=-\frac{\pi}{4} \frac{l}{R} \frac{\left|\rho_{i}-\rho_{e}\right|}{\rho_{i}+\rho_{e}} \omega_{k}
$$

This is equation $79 \mathrm{~b}$ of Goossens et al. (1992). Now rewrite (4.9) in terms of the damping time $\tau_{d}$ and the period to find

$$
\frac{\tau_{d}}{\text { Period }}=\frac{4}{\pi^{2}} \frac{R}{l} \frac{\rho_{i}+\rho_{e}}{\rho_{i}-\rho_{e}}
$$

which is (1.5). This is the result that we have obtained in Section 1 from equation 69 of Hollweg \& Yang (1988) by replacing the planar geometry into cylindrical geometry.

\section{Revival of damping of kink quasi-mode by resonant absorption}

\subsection{Proof of principle}

Ruderman \& Roberts (2002), slightly ahead of Goossens et al. (2002), were the first to study the damped kink quasi-mode in explicit relation to the damped oscillations seen in the TRACE observations. In the same way as Goossens et al. (1992) they confined the inhomogeneity to the interval

$$
b=R-l / 2 \leqslant r \leqslant a=R+l / 2
$$

They used the TB approximation and the TT approximation, but instead of a linear profile for the variation of density in the transitional layer, they adopted a sinusoidal profile. Their result for the damping time $\tau_{d}$ is

$$
\frac{\tau_{d}}{\text { Period }}=\frac{2}{\pi} \frac{R}{l} \frac{\rho_{i}+\rho_{e}}{\rho_{i}-\rho_{e}}
$$

This expression is remarkably similar to (1.5), the only difference being that the factor $4 /\left(\pi^{2}\right)$ in (1.5) is replaced with the factor $2 / \pi$ in $(5.1)$. The aim of Ruderman \& Roberts (2002) was to give a proof of the principle that resonant damping can explain the observed rapid damping. They took as an example period $\approx 300 \mathrm{sec}, \tau_{d} \approx 900 \mathrm{sec}$ and $\frac{\rho_{i}+\rho_{e}}{\rho_{i}-\rho_{e}} \approx 1$ and found that $l R \approx 0.2$. Ruderman \& Roberts (2002) concluded on the basis of their analysis and this example that the observed damping of coronal loop oscillations can be explained as damping of quasi-mode due to resonant absorption. A particular attraction 
of quasi-mode resonant damping is that it is fully consistent with the current estimates of very large coronal Reynolds numbers.

\subsection{Seismology $I I$}

Goossens et al. (2002) used the data on damping times of 11 loop oscillation events in order to estimate radial inhomogeneity length scales. Again the estimates for the density and in particular the density contrast $\rho_{i} / \rho_{e}$ are the weak link. Goossens et al. (2002) take $\rho_{i} / \rho_{e}=10$ and use the TTTB approximation (5.1) to estimate $l / R$ for 11 loops. They find values of $l / R$ in the range 0.16 to 0.49 . Goossens et al. (2002) confirm the conclusion by Ruderman \& Roberts (2002) and by Hollweg \& Yang (1988) that damped quasi-modes give an excellent explanation of the observed fast decay of oscillating loops if the inhomogeneity length scale is allowed to vary from loop to loop. The values found for $l / R$ by Goossens et al. (2002) are not entirely consistent with the assumption of a thin boundary layer adopted by Hollweg \& Yang (1988), Goossens et al. (1992), Ruderman \& Roberts (2002) and Goossens et al. (2002). There was an obvious need to relax the assumption of a thin boundary layer and to compute eigenmodes of fully non-uniform loops. A first attempt in this direction was made by Hollweg (1990), again in the context of coronal heating. Hollweg (1990) used the width of the resonance curves to estimate the free decay times of undrive surface quasi-modes. Van Doorsselaere et al. (2004) were the first to address the eigenvalue problem to determine numerically periods and damping times of quasi-modes in fully non-unform 1-dimensional equilibrium models.

\subsection{Damped kink quasi-modes for fully non-uniform 1-d loop models}

The complex eigenvalue problem based on the linear resistive MHD equations is solved numerically with the use of the eigenvalue code LEDA originally developed by van der Linden (1991). The first investigators to use LEDA in the context of coronal loop oscillations were Van Doorsselaere et al. (2004). With results for fully non-uniform oscillating loops available, it was possible to determine how accurate the TTTB results are. It turned out that the TTTB results are accurate with errors for the normalized damping rate not exceeding $5 \%$ for $l / R<0.4$. Hence this asymptotic formula is accurate far beyond its domain of validity. Note that for a fully non-uniform loop $l / R=2$. Caution is necessary when linking linear relations for the normalized damping rate in terms of $l / R, l / a$ and $l / b$. Recall that Goossens et al. (1992), Ruderman \& Roberts (2002) confine the inhomogeneity to the interval $b=R-l / 2 \leqslant r \leqslant a=R+l / 2$. When $l / R<<1$ it follows that $b \approx R \approx a$. However when $l / R \approx 1$ a linear relation in $l / R$ is no longer a linear relation in $l / a$ or $l / b$. As consequence analytical results that are equivalent for thin layers give rather differing results when extended to thick layers.

\subsection{Seismology III}

Aschwanden et al. (2003) used the observational info on damping times for 11 loops. They used the numerical results of Van Doorsselaere et al. (2004). Aschwanden et al. (2003) focussed on the density contrast $\zeta=\rho_{i} / \rho_{e}$. The aim of Aschwanden et al. (2003) was to compare values of $\zeta$ derived from observations of oscillating loops with values of that quantity determined by forward fitting of the cross-sectional density profile and line-of-sight integration of the cross-sectional fluxes observed with TRACE $171 \AA$. The part of this method that is related to the oscillations can be illustrated as follows. It is straightforward to rewrite the TTTB expression for the decay time (5.1) as

$$
\frac{\tau_{d}}{\text { Period }}=\frac{2}{\pi} \frac{R}{l} \frac{\zeta+1}{\zeta-1}
$$


The idea is then to observe $\tau_{d}$ /Period, to estimate $l / R$ and then to compute the density contrast $\zeta$. Recall that (5.2) is for thin non-uniform layers while there is strong suggestion that the non-uniform layers are thick. However, there is no need to use (5.2) since

$$
\frac{\text { Period }}{\tau_{d}}=f_{2}(\zeta, l / R)
$$

with $f_{2}(\zeta, l / R)$ a function that can be determined numerically as we shall explain in more detail in the following section.

Aschwanden et al. (2003) determined cross-sectional density profiles from observed emission measures (EM) and find values for $l / R, \zeta$. The loops turn out to be are fully non-uniform with $l / R=2$. Aschwanden et al. (2003) inverted the LEDA results (5.3) to obtain $\zeta$ from observed values of Period $/ \tau_{d}$ Comparison of the values of $\zeta$ determined from the oscillation data $\zeta_{\mathrm{LEDA}}$ with those obtained from forward modelling $\zeta_{\mathrm{EM}}$ leads to $\zeta_{\mathrm{LEDA}} / \zeta_{\mathrm{EM}} \approx 0.3 \cdots 0.8$. It is not clear where the differences come from. Probably both methods are inaccurate to some extent. Anyway, note that again only half of the observational info is used.

\section{Seismology of coronal loop oscillations}

So far we have referred to the seismological studies on TRACE observations of coronal loop oscillations by Nakariakov (2000) and Nakariakov \& Ofman (2001), Goossens et al. (2002) and Aschwanden et al. (2003). These studies only used part of the observational information. Nakariakov (2000) and Nakariakov \& Ofman (2001) used the observed periods to derive estimates for the strength of the magnetic field. The weak link in their analysis is the uncertainties on the density. Goossens et al. (2002) used the observed damping rates to derive estimates for the radial inhomogeneity length scale. Again, the weak link is the uncertainties on the density. Aschwanden et al. (2003) used the observed damping rates to determine the density contrast. The first study that used the observational information on both periods and damping times in the context of resonant damping in a consistent manner is by Arregui et al. (2007). Arregui et al. (2007) use the same 1-d cylindrical equilibrium models and the same numerical code LEDA as Van Doorsselaere et al. (2004).

The equilibrium models are characterized by the quantities: radius $R$, length $L$, density on axis $\rho_{i}$, density contrast $\zeta=\rho_{i} / \rho_{e}$, length of the radial inhomogeneous layer $l / R$ and the strength of the constant magnetic field $B$. The MHD waves are characterized by the wave numbers $m=1, k_{z}=\pi / L$, the real part of the frequency $\omega_{R}$ (or Period) and the damping time $\tau_{d}$. The internal Alfvén velocity $v_{A, i}$ and the the Alfvén travel time $\tau_{A, i}$ are defined as $v_{A, i}=B /\left(\sqrt{\mu \rho_{i}}\right), \quad \tau_{A, i}=L / v_{A, i}$. The relevant dimensionless quantities are

$$
\begin{gathered}
\zeta=\rho_{i} / \rho_{e}, \frac{l}{R}, \quad k_{z}^{\star}=k_{z} R=\pi R / L \\
\omega_{R}^{\star}=\omega_{R} \tau_{A i}, \text { Period }^{\star}=\operatorname{Period} / \tau_{A i} \tau_{d}^{\star}=\tau_{d} / \tau_{A i}
\end{gathered}
$$

The dimensionless wave number, frequency, period and damping time are indicated by $\star$. The input for the eigenvalue calculations with LEDA are values for the dimensionless quantities given in (6.1). The output are the corresponding results for the dimensionless quantities given in (6.2). Hence we have the following functional relations

$$
\operatorname{Period}^{\star}=\tilde{f}_{1}\left(k_{z}^{\star}, \zeta, l / R\right), \quad \tau_{d}^{\star}=\tilde{f}_{2}\left(k_{z}^{\star}, \zeta, l / R\right) .
$$

The functions $\tilde{f}_{1}$ and $\tilde{f}_{2}$ are to be determined numerically which requires computation of 
eigenvalues for a sufficiently large domain in the relevant 4-dimensional parameter space. If $R$ and $L$ are known with sufficient accuracy so that $k_{z}^{\star}=\pi R / L$ can be considered as known, (6.3) can be simplified to

$$
\frac{\text { Period }}{\tau_{A i}}=f_{1}(\zeta, l / R), \quad \frac{\text { Period }}{\tau_{d}}=f_{2}(\zeta, l / R)
$$

where now the functions $f_{1}$ and $f_{2}$ are to be determined numerically again with computation of eigenvalues for a sufficiently large domain now in the relevant 3-dimensional parameter space. Equations (6.4) contain two quantities that can be determined by observation, namely Period $\mathrm{d}_{o b s}$ and $\left(\operatorname{Period} /\left(\tau_{d}\right)\right)_{\text {obs }}$. They contain three unknown theoretical quantities, namely $\zeta, l / R$ and $\tau_{A i}$. Hence we have two equations for three unknown quantities

$$
\operatorname{Period}_{o b s}=\tau_{A i} f_{1}(\zeta, l / R), \quad\left(\frac{\text { Period }}{\tau_{d}}\right)_{o b s}=f_{2}(\zeta, l / R)
$$

As a consequence there are infinitely many equilibrium models that reproduce the observed period and damping time of a given oscillation event. In the 3-dimensional space $\left(\zeta, l / R, \tau_{A i}\right)$ the solutions define a 1-dimensional curve. This is illustrated in Fig. 3 of Arregui et al. (2007) where the solution curves for two oscillation events are shown. The conclusion is that the observed values of the period and the damping time do not allow a unique determination of even a 1-dimensional equilibrium model. However it turns out that the range of allowable values of $\tau_{A i}$ is rather restricted with a variation of typically $10 \%$.

\section{Conclusions and outlook}

The prediction by Hollweg \& Yang (1988), which was well well hidden in their paper, is correct. Resonant absorption can explain the fast damping of the loop oscillations observed by TRACE. The resonant damping requires thick non-uniform layers and relatively low density contrasts. For single mode oscillating loops the consistent use of the available information on periods and damping times shows that in the context of resonant damping of kink quasi-modes infinitely many 1-dimensional cylindrical equilibrium models can reproduce the observations. As it turns out the range in allowable Alfvén velocities on axis is small for most oscillation events putting definite constraints on this quantity. The theoretical studies on damped quasi-modes that this review has reported on are for 1-dimensional equilibrium models. Extensions to 2-dimensional equilibrium models are due to Andries et al. (2005a), Arregui et al. (2005), MacEwan et al. (2006), Dymova \& Ruderman (2005), Dymova \& Ruderman (2006). These studies have been triggered by the detection of double mode oscillating loops in a pioneering paper by Verwichte et al. (2004) and later confirmed by Van Doorsselaere et al. (2007). In particular the deviation of the ratio of the period of the fundamental mode to that of the first overtone from the canonical value of 2 has become a tool for investigating the longitudinal variation of density (see e.g Andries et al. (2005b), Goossens et al. (2006), Dymova \& Ruderman (2006), MacEwan et al. (2006).) In addition to seismology in the time domain using periods and damping times the detection of longitudinal stratification invites studies in the spatial domain focussing on the eigenfunctions (see e.g. Verth (2007), Erdélyi \& Verth (2007), Verth et al. (2007)). I am pretty sure MHD waves and coronal seismology will continue to intrigue us for at least the following two decades.

\section{Acknowledgements}

This review was written during a sabbatical leave. It is a pleasure to thank the FWOVlaanderen for their financial support. Over the years I have enjoyed and benefitted from 
collaborations and discussions with many colleagues. It was great fun. Special thanks go to J.V. Hollweg, M.S. Ruderman and T. Sakurai.

\section{References}

Andries, J., Tirry, W., Goossens, M. 2000, ApJ 531, 561

Andries, J., Goossens, M. 2001, A\&̊ 368, 1083

Andries, J., Goossens, M. 2001, A\&A 375, 1100

Andries, J., Goossens, M., Hollweg, J.V., Arregui, I., Van Doorsselaere, T. 2005, A\&A 430, 1109

Andries, J., Arregui, I., Goossens, M. 2005, ApJ 624, L57

Appert, K., Gruber, K., Vaclavik, J. 1974, Phys. Fluids 17, 1471

Arregui, I., Van Doorsselaere, T., Andries, J., Goossens, M., Kimpe, D. 2005, A\&BA 441, 361

Arregui, I., Andries, J., Van Doorsselaere, T., Goossens, M., Poedts, S. 2007, A\&3A 463, 333

Ashwanden, M.J., Fletcher, L., Schrijver, C.J.,Alexander, D. 1999, ApJ 520, 880

Ashwanden, M.J., Nightingale, R., Andries, J., Van Doorsselaere, T., Goossens, M. 2003, ApJ 598,1375

Cally, P.S. 1986, Sol. Phys. 103, 277

Chen, L., Hasegawa, A. 1974, Phys. Fluids 17, 1399

Dymova, M.V., Ruderman, M.S. 2005, Sol. Phys. 229, 79

Dymova, M.V., Ruderman, M.S. 2006, A $\mathscr{E} A$ 459, 241

Edwin, P., Roberts, B.R. 1983, Sol. Phys. 88, 179

Erdélyi, R. 1997, Sol.Phys 171, 49

Erdélyi, R. \& Fedun, V. 2006, Solar Physics, 238, 41

Erdélyi, R. \& Fedun, V. 2007, Solar Physics, 246, 101

Erdélyi, R. \& Goossens, M. 1996, A\& $A, 294,575$

Erdélyi, R. \& Goossens, M. 1996, A\& $A, 313,664$

Erdélyi, R., Goossens, M. \& Ruderman, M.S. 1995, Sol.Phys 161, 123

Erdélyi R. \& Taroyan 2003, JGR 108, 1043

Erdélyi, R. \& Verth, G. 2007, A\&A, 462, 743

Goedbloed, J.P. 1983, Lecture Notes on Ideal Magnetohydrodynamics, Rijnhuizen Report 83-145.

Goossens, M., Hollweg, J.V., Sakurai, T. 1992, Sol.Phys 138, 233

Goossens, M., Hollweg, J.V. 1993, Sol. Phys. 145, 19

Goossens, M., Ruderman, M.S., Hollweg, J.V. 1995, Sol.Phys 157, 75

Goossens, M., Ruderman, M.S. 1995, Physica Scripta T60, 171

Goossens, M., Andries, J., Aschwanden, M.J. 2002, A\&A 394, L39

Goossens, M., Andries, J., Arregui, I. 2006, Roy. Soc. London Philos. Trans. Ser. A 364, 433

Hollweg, J.V. \& Yang, G. 1988, J. Geophys. Res. 93, 5423

Hollweg, J.V. 1990, J. Geophys. Res. 95, 2319

Kappraff, J.M. \& Tataronis, J. 1977, J. Plasma Physics 18, 209

MacEwan, M.P., Donnelly, G.R., Diaz, A.J., Roberts, B.R. 2006, A $\& A 460,893$

Nakariakov, V.M., Ofman, L., DeLuca, E.E., Roberts, B.R., Davila, J.M. 1999, Science 285, 862

Nakariakov, V.M. 2000, AIP CP537, 264

Nakariakov, V.M., Ofman, L. 2001, A\&A 372, L53

Pintér, B., Erdélyi, R., Goossens, M. 2007, A\& $A$ 466, 377

Poedts, S., Goossens, M., Kerner, W. 1989, Sol. Phys 123, 83

Poedts, S., Goossens, M., Kerner, W. 1990, ApJ 360, 279

Poedts, S., Kerner, W. 2001, Physical Review Letters 66, 2871

Ruderman, M.S., Roberts, B.R. 2002, ApJ 577, 475

Sakurai, T., Goossens, M., Hollweg, J.V. 1991, Sol.Phys 133, 227

Sakurai, T., Goossens, M., Hollweg, J.V. 1991, Sol.Phys 133, 247

Spruit, H.C. 1982, Sol. Phys. 75, 3

Stenuit, H., Erdélyi, R., Goossens, M. 1995, Sol. Phys. 161, 139

Stenuit, H., Keppens, R., Goossens, M. 1998, A $\mathscr{E} A$ 331, 392 
Stenuit, H., Tirry, W.J., Keppens, R., Goossens, M. 1999, A $E A$ 342, 863

Taroyan, Y. \& Erdélyi R. 2002, Phys. Plas. 9, 312

Taroyan, Y. \& Erdélyi R. 2003, JGR 108, 1301

Tirry, W.J, Goossens, M. 1995, J. Geophys. Res. 100, 23687

Tirry, W.J, Goossens, M. 1996, ApJ 471, 501

Tirry, W.J, Cadez, V., Erdélyi, R. \& Goossens 1998a, A\&A A332, 786

Tirry, W.J, Goossens, M., Pintér, B., Cadez, V., Vanlommel, P. 1998b, ApJ 503, 422

Terradas, J., Oliver, R., Ballester, J.L. 2006, ApJ 642, 540

Terradas, J., Andries, J. Goossens, M. 2007, A\& $\mathcal{E}$ 469, 1135

van der Linden, R.A.M. 1991, Ph. D. Thesis K.U.Leuven

Van Doorsselaere, T., Andries, J., Poedts, D., Goossens, M. 2004, ApJ 606, 1223

Van Doorsselaere, T., Nakariakov, V.M., Verwichte, E. 2007, $A \mathscr{E} A$

Verth, G. 2007, Astron. Nachr. 328, 764

Verth, G., Van Doorsselaere, T., Erdélyi, R., Goossens, M. 2007, A\&̈A, 475, 341

Verwichte, E., Nakariakov, V.M., Ofman, L., Deluca, E.E. 2004, Sol. Phys. 223, 77 